\title{
Planar Hamiltonian systems at resonance: the Ahmad-Lazer-Paul condition
}

\author{
Alberto Boscaggin and Maurizio Garrione
}

\begin{abstract}
We consider the planar Hamiltonian system

$$
J u^{\prime}=\nabla F(u)+\nabla_{u} R(t, u), \quad t \in[0, T], u \in \mathbb{R}^{2},
$$

with $F(u)$ positive and positively 2-homogeneous and $\nabla_{u} R(t, u)$ sublinear in $u$. By means of an Ahmad-Lazer-Paul type condition, we prove the existence of a $T$-periodic solution when the system is at resonance. The proof exploits a symplectic change of coordinates which transforms the problem into a perturbation of a linear one. The relationship with the Landesman-Lazer condition is analyzed, as well.
\end{abstract}

Mathematics Subject Classification (2010). Primary 34C25; Secondary 37J45.

Keywords. Periodic solutions, Resonance, Hamiltonian systems,

Ahmad-Lazer-Paul condition, Symplectic maps.

\section{Introduction and statement of the main result}

For the scalar second order differential equation

$$
x^{\prime \prime}+\lambda_{k} x+w(t, x)=0, \quad x \in \mathbb{R},
$$

being $w:[0, T] \times \mathbb{R} \rightarrow \mathbb{R}$ a continuous and bounded function and $\lambda_{k}=\left(\frac{2 k \pi}{T}\right)^{2}$ $(k=0,1, \ldots)$, a classical issue is represented by the existence of $T$-periodic solutions. Indeed, since $\lambda_{k}$ is an eigenvalue of the linear differential operator $x \mapsto-x^{\prime \prime}$ with $T$-periodic boundary conditions, equation (1.1) is at resonance, and no $T$-periodic solutions in general exist.

In [23, Theorem 4.8 and Exercise 4.9], an existence result was proved-by variational tools - under the assumption

$$
\lim _{\substack{\|x\| \infty \rightarrow+\infty \\ x^{\prime \prime}+\lambda_{k} x=0}} \int_{0}^{T} W(t, x(t)) d t=+\infty,
$$

With our warmest wishes to Professor Fabio Zanolin for his 60th birthday. 
being $W(t, x)=\int_{0}^{x} w(t, \xi) d \xi$. Condition (1.2) is usually referred to as the Ahmad-Lazer-Paul condition, since it is the version, for the $T$-periodic problem, of the assumption introduced in [1], dealing with the Dirichlet problem for an elliptic partial differential equation at resonance. Qualitatively, (1.2) expresses the anticoercivity of the Lagrange functional associated with (1.1) on the eigenspace relative to $\lambda_{k}$, so that a $T$-periodic solution can be provided as a critical point of saddle type. We remark that the result in [23] holds as well for second order systems of gradient type

$$
x^{\prime \prime}+\lambda_{k} x+\nabla_{x} W(t, x)=0, \quad x \in \mathbb{R}^{N} ;
$$

further developments along this direction were obtained, among the others, in $[3,16,27]$.

Later, related results $[6,15,19,22,26]$ were given for general Hamiltonian systems of the type

$$
J u^{\prime}=A(t) u+\nabla_{u} R(t, u), \quad u \in \mathbb{R}^{2 N},
$$

where $J=\left(\begin{array}{cc}0 & -I_{N} \\ I_{N} & 0\end{array}\right)$ is the standard symplectic matrix, $A(t)$ is a continuous path of $2 N \times 2 N$ symmetric matrices and $R:[0, T] \times \mathbb{R}^{2 N} \rightarrow \mathbb{R}$ is a regular function with bounded gradient. Resonance is here meant in the sense that the linear problem $J u^{\prime}=A(t) u$ has nontrivial $T$-periodic solutions. Notice that, in this case, more sophisticated techniques from critical point theory are needed, since the natural variational formulation of (1.3) leads to a strongly indefinite functional (i.e., its quadratic part is unbounded both from below and from above).

On the other hand, starting with the pioneering works $[5,14]$, a growing attention has been devoted to the scalar second order equation

$$
x^{\prime \prime}+\mu x^{+}-\nu x^{-}+w(t, x)=0, \quad x \in \mathbb{R},
$$

with $x^{+}=\max \{x, 0\}, x^{-}=\max \{-x, 0\}$, where $w:[0, T] \times \mathbb{R} \rightarrow \mathbb{R}$ is continuous and bounded and $(\mu, \nu)$ belongs to the $T$-periodic Dancer-Fučik spectrum, i.e., $\mu, \nu>0$ and, for a positive integer $N$,

$$
\frac{\pi}{\sqrt{\mu}}+\frac{\pi}{\sqrt{\nu}}=\frac{T}{N} .
$$

As well known, such a concept of asymmetric resonance extends the linear one $\mu=\nu=\lambda_{N}$. As for the existence of $T$-periodic solutions to (1.4) via Ahmad-Lazer-Paul type conditions, the problem is more subtle, because the asymmetry of the unperturbed problem avoids the use of the linear tools usually employed to detect a saddle geometry (see [25]). In this connection, some results were given in $[4,18]$.

In this paper, we are concerned with Ahmad-Lazer-Paul type conditions for planar Hamiltonian systems of the type

$$
J u^{\prime}=\nabla F(u)+\nabla_{u} R(t, u), \quad u \in \mathbb{R}^{2},
$$


where $J=\left(\begin{array}{cc}0 & -1 \\ 1 & 0\end{array}\right), R:[0, T] \times \mathbb{R}^{2} \rightarrow \mathbb{R}$ and $F: \mathbb{R}^{2} \rightarrow \mathbb{R}$ is positive and positively 2-homogeneous, i.e.,

$$
0<F(\lambda u)=\lambda^{2} F(u), \quad \text { for every } u \neq 0 \text { and } \lambda>0 .
$$

This setting was introduced in [9] as a natural extension of the asymmetric equation (1.4): indeed, for $u=(x, y)$, it suffices to set $F(u)=\frac{1}{2}\left(\mu\left(x^{+}\right)^{2}+\right.$ $\left.\nu\left(x^{-}\right)^{2}+y^{2}\right)$ and $R(t, u)=(W(t, x), 0)$. Further developments were given, for instance, in $[8,12]$. Under assumption (1.7), the origin is an isochronous center for the autonomous Hamiltonian system

$$
J u^{\prime}=\nabla F(u),
$$

namely all the nontrivial solutions to (1.8) are periodic with the same minimal period $\tau$, so that resonance appears if and only if

$$
\tau=\frac{T}{N},
$$

for a positive integer $N$. As proved in [9], when (1.9) is verified, (1.6) may not have $T$-periodic solutions.

Our aim is to provide an Ahmad-Lazer-Paul condition (see (1.11) below) to ensure existence also in this resonant setting. Actually, on the lines of $[6,15$, 27], $\nabla_{u} R(t, u)$ does not need to be bounded (cf. Remark 3.1), but is allowed to grow at infinity as a sublinear power (in the $u$-variable). Precisely, here is the statement of our main result.

Theorem 1.1. Let $F \in C^{1}\left(\mathbb{R}^{2}\right) \cap C^{2}\left(\mathbb{R}^{2} \backslash\{0\}\right)$ satisfy (1.7) and (1.9), and let $R \in C^{1}\left([0, T] \times \mathbb{R}^{2}\right)$ fulfill, for suitable constants $M>0, \alpha \in[0,1[$, the growth condition

$$
\left|\nabla_{u} R(t, u)\right| \leq M\left(1+|u|^{\alpha}\right), \quad \text { for every } t \in[0, T], u \in \mathbb{R}^{2} .
$$

Moreover, denoting by $\varphi(t)$ the solution to $(1.8)$ such that $\varphi(0)=(1,0)$, suppose that

$$
\lim _{\lambda \rightarrow+\infty} \frac{1}{\lambda^{2 \alpha}} \int_{0}^{T} R(t, \lambda \varphi(t+\theta)) d t=+\infty, \quad \text { uniformly in } \theta \in\left[0, \frac{T}{N}[.\right.
$$

Then, system (1.6) has a T-periodic solution.

Recall that, in view of the homogeneity of $F(u)$, the family

$$
\left\{\lambda \varphi(\cdot+\theta) \mid \lambda>0, \theta \in\left[0, \frac{T}{N}[\}\right.\right.
$$

gives exactly the set of the nontrivial solutions to (1.8). The strategy of the proof of Theorem 1.1 is inspired by the arguments in [13,18,19], exploiting a symplectic change of coordinates which tranforms the original system (1.6) into a (Hamiltonian) perturbation of a linear one, like (1.3). The conclusion is then achieved using variational tools, following [15].

For the asymmetric equation (1.4), we get the following corollary, improving [4] (where $w(t, x)$ is bounded and $(\mu, \nu)$ is "not too far" from the diagonal) and [18] (where $w(t, x)$ is bounded; cf. Remark 3.2). 
Corollary 1.2. Let $\mu, \nu>0$ satisfy (1.5) and let $w \in C([0, T] \times \mathbb{R})$ fulfill, for suitable constants $M>0, \alpha \in[0,1[$, the growth condition

$$
|w(t, x)| \leq M\left(1+|x|^{\alpha}\right), \quad \text { for every } t \in[0, T], x \in \mathbb{R} .
$$

Moreover, setting

$$
\phi(t)= \begin{cases}\frac{1}{\sqrt{\mu}} \sin (\sqrt{\mu} t) & \text { if } t \in\left[0, \frac{\pi}{\sqrt{\mu}}\right] \\ \frac{1}{\sqrt{\nu}} \sin \left(\sqrt{\nu}\left(\frac{\pi}{\sqrt{\mu}}-t\right)\right) & \text { if } t \in\left[\frac{\pi}{\sqrt{\mu}}, \frac{T}{N}\right],\end{cases}
$$

and still denoting by $\phi(t)$ its $\frac{T}{N}$-periodic extension, suppose that

$$
\lim _{\lambda \rightarrow+\infty} \frac{1}{\lambda^{2 \alpha}} \int_{0}^{T} W(t, \lambda \phi(t+\theta)) d t=+\infty, \quad \text { unif. in } \theta \in\left[0, \frac{T}{N}[,\right.
$$

being $W(t, x)=\int_{0}^{x} w(t, \xi) d \xi$. Then, equation (1.4) has a T-periodic solution.

The plan of the article is as follows. In Sect. 2, we describe in detail the symplectic change of variables which will be used in the proof of Theorem 1.1 and we briefly recall the variational framework to treat the $T$-periodic problem associated with (1.3). Section 3 is devoted to the proof of Theorem 1.1. In Sect. 4, we compare our Ahmad-Lazer-Paul condition with another nonresonance assumption, coming from topological degree theory: the Landesman-Lazer one (see [20], for the Dirichlet problem in the PDE setting). In the framework of system (1.6), such a condition was recently given in [10], as a generalization of the ones introduced in [21] for (1.1) and in [7] for (1.4). On the lines of [11], we show that the Ahmad-Lazer-Paul condition is implied by the Landesman-Lazer one. Lastly, some technical details are discussed in a brief Appendix.

Notation and terminology. In the following, $\mathbb{R}^{2}$ will denote the plane, with Euclidean scalar product $\langle\cdot \mid \cdot\rangle$ and Euclidean norm $|\cdot|$, whereas, by $\mathbb{R}_{*}^{2}$, we will mean the punctured plane $\mathbb{R}^{2} \backslash\{0\}$. Similarly, by $\mathbb{R}_{*}^{+}$we will denote the set of (strictly) positive real numbers. For a $2 \times 2$ square matrix $A$, we will write $A^{t}$ for its transpose and $\|A\|$ for its operatorial norm. If $\Lambda: \mathcal{U} \rightarrow \mathbb{R}^{2}$ is a $C^{1}$-map, being $\mathcal{U} \subset \mathbb{R}^{2}$ an open set, $\Lambda^{\prime}(u)$ will be the Jacobian matrix of $\Lambda$, evaluated at the point $u \in \mathcal{U}$. Lastly, for a function $V$ depending on time and space, we will briefly write $\nabla V$ to denote the gradient of $V$ with respect to its space variable.

By a $T$-periodic solution to (1.6) we will mean, as usual, a solution $u(t)$ satisfying the boundary condition $u(0)=u(T)$. Indeed, whenever $R(t, u)$ is defined for every $t \in \mathbb{R}$, with $R(\cdot, u) \equiv R(\cdot+T, u)$ for every $u \in \mathbb{R}^{2}$, every solution to (1.6) defined on $[0, T]$ and satisfying $u(0)=u(T)$ can be extended, by $T$-periodicity, to a solution on the whole real line.

\section{Preliminaries}

In this section, we collect some preliminary tools, which will be employed throughout Sect. 3 . 


\subsection{A symplectic transformation}

Recall that, for an open set $\mathcal{U} \subset \mathbb{R}^{2}$, a $C^{1}$-map $\Lambda: \mathcal{U} \rightarrow \mathbb{R}^{2}$ is called symplectic if

$$
\Lambda^{\prime}(u)^{t} J \Lambda^{\prime}(u)=J, \quad \text { for every } u \in \mathcal{U} .
$$

It is well known that, given a $C^{1}$-function $V:[0, T] \times \mathbb{R}^{2} \rightarrow \mathbb{R}$ and a symplectic $C^{1}$-diffeomorphism $\Lambda$ of the plane onto itself, the Hamiltonian system $J u^{\prime}=\nabla V(t, u)$ is changed, via the change of variables $v=\Lambda(u)$, into the system $J v^{\prime}=\nabla \widetilde{V}(t, v)$, being $\widetilde{V}(t, v)=V\left(t, \Lambda^{-1}(v)\right)$. This means that the transformed system is still Hamiltonian, and the associated Hamiltonian is just the "old" one, evaluated on the "new" variable $v$.

We are going to construct a symplectic $C^{1}$-diffeomorphism of the plane, in association with a nonnegative function $H: \mathbb{R}^{2} \rightarrow \mathbb{R}$ satisfying the following hypotheses:

(H0) $H \in C^{1}\left(\mathbb{R}^{2}\right) \cap C^{2}\left(\mathbb{R}_{*}^{2}\right)$;

(H1) $H(0)=0$ and $H(u)>0$ for every $u \neq 0$;

(H2) $\nabla H(0)=0$ and $\langle\nabla H(u) \mid u\rangle>0$ for every $u \neq 0$;

(H3) $H(u) \rightarrow+\infty$ for $|u| \rightarrow+\infty$.

Notice that, in view of Euler's formula, the functions of class $C^{1}\left(\mathbb{R}^{2}\right) \cap$ $C^{2}\left(\mathbb{R}_{*}^{2}\right)$ satisfying (1.7) fulfill (H1)-(H3).

Under the above hypotheses, it is possible to well describe the dynamics of the planar autonomous Hamiltonian system

$$
J u^{\prime}=\nabla H(u)
$$

as we are going to show in the following lemma.

Lemma 2.1. Assume (H0)-(H3). Then, there is uniqueness and global continuability for the solutions to the Cauchy problems associated with (2.2). Moreover, every nontrivial solution to (2.2) is periodic and its orbit is a strictly star-shaped Jordan curve around the origin, covered in the clockwise sense.

Proof. The uniqueness for an initial datum different from zero follows from the fact that $\nabla H(u)$ is locally Lipschitz continuous on $\mathbb{R}_{*}^{2}$. On the other hand, nontrivial solutions never reach the origin, in view of the preservation of $H(u)$ and (H1). Setting, for $c>0$,

$$
\Gamma_{c}=\left\{u \in \mathbb{R}^{2} \mid H(u)=c\right\},
$$

we claim that $\Gamma_{c}$ is a strictly star-shaped Jordan curve around the origin, i.e., for every $\xi \in \mathbb{S}^{1}$, the ray emanating from the origin and passing through $\xi$ intersects $\Gamma_{c}$ exactly once. This follows from the fact that the map $\gamma: \lambda \in$ $\mathbb{R}_{*}^{+} \mapsto H(\lambda \xi)$ is strictly increasing (by (H2)), and such that (by (H1) and (H3))

$$
\lim _{\lambda \rightarrow 0^{+}} \gamma(\lambda)=0, \quad \lim _{\lambda \rightarrow+\infty} \gamma(\lambda)=+\infty
$$

Since, by the preservation of the energy, every solution $u(t)$ to $(2.2)$ lies on a level curve of $H(u), u(t)$ is globally defined and periodic. Finally, writing 
$u(t)=\rho(t)(\cos \omega(t), \sin \omega(t))$, with $\rho(t)>0$, a standard computation gives

$$
-\omega^{\prime}(t)=\frac{\left\langle J u^{\prime}(t) \mid u(t)\right\rangle}{|u(t)|^{2}}=\frac{\langle\nabla H(u(t)) \mid u(t)\rangle}{|u(t)|^{2}}>0,
$$

so that $u(t)$ moves clockwise.

In this setting, it is said that the origin is a global center for system (2.2); for every $u \neq 0$, we denote by $\tau(u)$ the minimal period of the solution passing through $u$. For further convenience, we underline that the map $u \mapsto \tau(u)$ is of class $C^{1}$ (see the Appendix).

After these preliminary considerations, we can state the following proposition, dealing with the case when the origin is an isochronous center (see $(2.3))$.

Proposition 2.2. Let $H(u)$ satisfy $(\mathrm{H} 0)-(\mathrm{H} 3)$. Assume that there exist $\tau, r>0$ such that

$$
\tau(u)=\tau, \quad \text { for every } u \neq 0,
$$

and

$$
H(u)=\frac{\pi}{\tau}|u|^{2}, \quad \text { for every }|u|<r .
$$

Then, there exists a symplectic $C^{1}$-diffeomorphism $\Lambda_{H}$ of $\mathbb{R}^{2}$ onto itself such that

$$
H\left(\Lambda_{H}^{-1}(v)\right)=\frac{\pi}{\tau}|v|^{2}, \quad \text { for every } v \in \mathbb{R}^{2} .
$$

Let us observe that, if (2.4) holds, all the solutions $u(t)$ to (2.2) such that $|u(t)|<r$ are of the type $\lambda\left(\cos \left(\frac{2 \pi}{\tau}(t+\theta)\right),-\sin \left(\frac{2 \pi}{\tau}(t+\theta)\right)\right)$, for suitable constants $\lambda, \theta>0$, so that they are periodic with minimal period equal to $\tau$. Hence, (2.3) and (2.4) are not contradictory.

Geometrically, (2.5) means that the level curves of $H(u)$ are transformed, through $\Lambda_{H}$, into circumferences around the origin, so that the nonlinear system (2.2) is changed into the linear one

$$
J v^{\prime}=\frac{2 \pi}{\tau} v .
$$

We now prove the proposition.

Proof. For every $u \neq 0$, consider the solution $\zeta(t, u)$ to $(2.2)$ such that $\zeta(0, u)=$ $u$ and define $\theta(u) \in[0,2 \pi[$ as the minimum time for which

$$
\zeta\left(-\frac{\tau}{2 \pi} \theta(u), u\right) \in \mathbb{R}_{*}^{+} \times\{0\} .
$$

To justify the forthcoming computations, we observe the following facts concerning the regularity of the map $u \mapsto \theta(u)$ :

(i) the map $\theta: \mathbb{R}_{*}^{2} \backslash\left(\mathbb{R}_{*}^{+} \times\{0\}\right) \rightarrow \mathbb{R}$ is of class $C^{1}$ and $\nabla \theta(u)$ extends to a continuous function (which we still denote by $\nabla \theta(u)$ ) on the whole $\mathbb{R}_{*}^{2}$; moreover, if $u(t)$ is a solution to $(2.2)$, it holds that 


$$
(\langle J \nabla \theta(u(t)) \mid \nabla H(u(t))\rangle=) \frac{d}{d t} \theta(u(t))=\frac{2 \pi}{\tau} ;
$$

(ii) the map $\mathbb{R}_{*}^{2} \ni u \mapsto(\cos \theta(u),-\sin \theta(u))$ is continuous.

All the assertions basically follow from the geometrical interpretation of $\theta(u)$ and the regularity of $\zeta(t, u)$, together with the Implicit Function Theorem. Notice that (i) and (ii) imply that $u \mapsto(\cos \theta(u),-\sin \theta(u))$ is of class $C^{1}$. For a discussion about these properties, see the Appendix.

We now define the map $\Lambda_{H}: \mathbb{R}_{*}^{2} \rightarrow \mathbb{R}^{2}$ as

$$
\Lambda_{H}(u)=\sqrt{\frac{\tau}{\pi} H(u)}(\cos \theta(u),-\sin \theta(u)) .
$$

In view of the previous considerations, $\Lambda_{H}$ is of class $C^{1}$; moreover, using (2.4), it is easy to see that $\Lambda_{H}(u)=u$ for every $|u|<r$, so that $\Lambda_{H}$ extends (setting $\left.\Lambda_{H}(0)=0\right)$ to a $C^{1}$ function on the whole $\mathbb{R}^{2}$, still denoted in the same way. Second, a simple calculation yields, in view of (2.6),

$$
\operatorname{det} \Lambda_{H}^{\prime}(u)=\frac{\tau}{2 \pi}\langle J \nabla \theta(u) \mid \nabla H(u)\rangle=1
$$

which, by a direct computation, implies (2.1). Third, the fact that $\Lambda_{H}$ is a $C^{1}$-diffeomorphism follows from the Hadamard-Caccioppoli global inversion Theorem. Indeed, (2.8) implies that $\Lambda_{H}$ is $C^{1}$-locally invertible; moreover, $\Lambda_{H}$ is also a proper map (i.e., the preimage of compact sets is compact), since $\left|\Lambda_{H}(u)\right| \rightarrow+\infty$ for $|u| \rightarrow+\infty$, in view of (H3).

Finally, relation (2.5) follows from (2.7) - just taking the modulus and setting $u=\Lambda_{H}^{-1}(v)$.

Remark 2.3. We stress that assumption (2.4) is needed to guarantee that $\Lambda_{H}$ is of class $C^{1}$ up to the origin; this is not the case for a general function $H(u)$ satisfying (2.3). To show this, consider for example a positive and positively 2-homogeneous function $H(u)$. In this case, it can be seen - noticing that $\theta(u)$ is positively 0-homogeneous - that $\Lambda_{H}$ is positively 1-homogeneous, so that $\Lambda_{H}^{\prime}$ is constant on every ray emanating from the origin. Accordingly, $\Lambda_{H}^{\prime}$ is not continuous, except when $\Lambda_{H}^{\prime}$ is constant, i.e., $\Lambda_{H}$ linear. However, $\Lambda_{H}(u)=A u$ for a square matrix $A$ implies, using (2.7),

$$
H(u)=\frac{\pi}{\tau}\left|\Lambda_{H}(u)\right|^{2}=\frac{\pi}{\tau}\left\langle A^{t} A u \mid u\right\rangle,
$$

namely $H(u)$ is a (positive definite) quadratic form, which is not in general the case. We finally remark that, when only $(2.3)$ is assumed, $\Lambda_{H}$ is a symplectic diffeomorphism of $\mathbb{R}_{*}^{2}$ onto itself, as it can be seen by slightly different arguments (see $[13,18]$ for a guideline).

\subsection{The variational setting}

We consider the planar Hamiltonian system

$$
J v^{\prime}=B(t) v+\nabla Z(t, v),
$$

being $B(t), t \in[0, T]$, a continuous path of symmetric $2 \times 2$ matrices and $Z:[0, T] \times \mathbb{R}^{2} \rightarrow \mathbb{R}$ a $C^{1}$-function. The $T$-periodic problem associated with 
(2.9) can be rephrased as a critical point problem, for a functional defined on a suitable Hilbert space, as follows.

We define

$$
E=\left\{v \in L^{2}(] 0,\left.T\left[; \mathbb{R}^{2}\right)\left|\sum_{k \in \mathbb{Z}}(1+|k|)\right| v_{k}\right|^{2}<+\infty\right\},
$$

being $\sum_{k \in \mathbb{Z}} e^{\frac{2 k \pi}{T} t J} v_{k}$, with $v_{k} \in \mathbb{R}^{2}$, the Fourier expansion of $v(t)$. The space $E$ is a fractional Sobolev space (usually denoted also by $H_{T}^{1 / 2}$ ) and has a structure of Hilbert space, endowed with the scalar product

$$
\langle v \mid w\rangle_{E}=\sum_{k \in \mathbb{Z}}(1+|k|)\left\langle v_{k} \mid w_{k}\right\rangle .
$$

Since, for $v, w$ smooth, the bilinear map

$$
(v, w) \mapsto \int_{0}^{T}\left\langle J v^{\prime}(t) \mid w(t)\right\rangle d t
$$

is continuous with respect to the norm of $E$, by density and the Riesz representation theorem there exists a unique linear bounded operator $\mathcal{L}: E \rightarrow E$ such that, for $v, w$ smooth,

$$
\langle\mathcal{L} v \mid w\rangle_{E}=\int_{0}^{T}\left\langle J v^{\prime}(t) \mid w(t)\right\rangle d t .
$$

Define, at this point, the functional

$$
\mathcal{I}(v)=\langle\mathcal{L} v \mid v\rangle_{E}-\int_{0}^{T}\langle B(t) v(t) \mid v(t)\rangle d t-\int_{0}^{T} Z(t, v(t)) d t, \quad v \in E .
$$

We notice that the first integral is well-defined in view of the embedding $E \hookrightarrow$ $L^{2}(] 0, T\left[; \mathbb{R}^{2}\right)$, whereas the second one is just formal, since $v(t)$ may not be continuous. However, we have the following proposition (see $[2,24])$.

Proposition 2.4. Assume that there exist $m>0, s \in] 2,+\infty[$ such that

$$
|\nabla Z(t, v)| \leq m\left(1+|v|^{s-1}\right), \quad \text { for every } t \in[0, T], v \in \mathbb{R}^{2} .
$$

Then, $\mathcal{I}: E \rightarrow \mathbb{R}$ is of class $C^{1}$ and its critical points are (classical) T-periodic solutions to (2.9).

Furthermore, when (2.9) is the perturbation of a linear problem at resonance, we have the following result (see [15, Theorem 1.1]), which was somehow already announced in [18, Remark, p. 1225].

Theorem 2.5. Denote by $\mathfrak{S}$ the set of the T-periodic solutions to $J v^{\prime}=B(t) v$ and assume $\mathfrak{S} \neq\{0\}$. Moreover, suppose that there exists $\widetilde{M}>0$ and $\alpha \in[0,1[$ such that 


$$
|\nabla Z(t, v)| \leq \widetilde{M}\left(1+|v|^{\alpha}\right), \quad \text { for every } t \in[0, T], \quad v \in \mathbb{R}^{2} .
$$

If

$$
\lim _{\substack{\|v\|_{E} \rightarrow+\infty \\ v \in \mathfrak{S}}} \frac{1}{\|v\|_{E}^{2 \alpha}} \int_{0}^{T} Z(t, v(t)) d t=+\infty
$$

then system (2.9) has a T-periodic solution.

Observe that $\mathfrak{S}$ is a linear subspace having finite dimension, so that any other norm on $\mathfrak{S}$ could be used in (2.12). In the following, for simplicity, we will use the $L^{\infty}$-norm.

For what concerns the proof of Theorem 2.5, observe first that (2.11) implies (2.10), so that (2.9) can be studied in the previously introduced variational setting. The assumptions (2.11) and (2.12) are then used to ensure the validity of the Palais-Smale condition and a saddle type geometry associated with an orthogonal decomposition $E=E_{1} \oplus E_{2}$. However, since both $E_{1}$ and $E_{2}$ are infinite dimensional, a finer version of Rabinowitz saddle point theorem needs to be used [24, Theorem 5.29 and Example 5.22].

\section{Proof of the main result and further remarks}

The main ingredient of the proof of Theorem 1.1 consists in transforming system (1.6), via the symplectic change of variables described in Sect. 2, into a perturbation of a linear one. However, since $F(u)$ does not satisfy (2.4) - unless $F(u)=\frac{\pi}{\tau}|u|^{2}$ for every $u \in \mathbb{R}^{2}$-we need the following preliminary trick.

Fix $0<r_{1}<r_{2}$, and $\epsilon>0$ such that

$$
\epsilon|u|^{2} \leq F(u), \quad \text { for every } r_{1} \leq|u| \leq r_{2} .
$$

Moreover, choose a regular nondecreasing function $\beta:[0,+\infty[\rightarrow \mathbb{R}$ such that

- $\beta(x)=0$ for every $x \leq r_{1}^{2}$ and $\beta(x)=1$ for every $x \geq r_{2}^{2}$;

- $\quad 0<\beta(x)<1$ for every $r_{1}^{2}<x<r_{2}^{2}$.

Now, define $G: \mathbb{R}^{2} \rightarrow \mathbb{R}$ as

$$
G(u)=\left(1-\beta\left(|u|^{2}\right)\right) \epsilon|u|^{2}+\beta\left(|u|^{2}\right) F(u) .
$$

We claim that $G(u)$ satisfies (H0)-(H3). Indeed, (H0), (H1) and (H3) are straightly proved, while (H2) follows, in view of (3.1), from the fact that

$$
\begin{aligned}
\langle\nabla G(u) \mid u\rangle= & 2 \beta^{\prime}\left(|u|^{2}\right)|u|^{2}\left[F(u)-\epsilon|u|^{2}\right]+2 \epsilon\left(1-\beta\left(|u|^{2}\right)\right)|u|^{2} \\
& +\beta\left(|u|^{2}\right)\langle\nabla F(u) \mid u\rangle .
\end{aligned}
$$

Hence, Lemma 2.1 implies that the origin is a center for $J u^{\prime}=\nabla G(u)$, and we denote by $\tau_{G}(u)$ the minimal period of the solutions passing through $u$. Since, in general, $\tau_{G}(u)$ is not constant, in order to apply Proposition 2.2 we need a further modification.

For $c \in \mathbb{R}_{*}^{+}$, let us define $\xi(c)$ as the unique positive number such that $G(\xi(c), 0)=c$, i.e. $(\xi(c), 0)$ is the intersection between the level curve $\left\{u \in \mathbb{R}^{2} \mid\right.$ 
$G(u)=c\}$ and the positive $x$-semiaxis; the map $c \mapsto \xi(c)$ is clearly continuous. We define

$$
H(u)=\frac{1}{\tau} \int_{0}^{G(u)} \tau_{G}(\xi(c), 0) d c
$$

by construction we have

$$
\nabla H(u)=\frac{\tau_{G}(\xi(G(u)), 0)}{\tau} \nabla G(u)=\frac{\tau_{G}(u)}{\tau} \nabla G(u) .
$$

Since, as remarked before Proposition 2.2, $u \mapsto \tau_{G}(u)$ is of class $C^{1}$ on $\mathbb{R}_{*}^{2}$, we have that $H(u)$ satisfies $(\mathrm{H} 0)$. On the other hand, (H1)-(H3) are easily proved and, by construction, all the nontrivial solutions to $J u^{\prime}=\nabla H(u)$ have minimal period $\tau$ (cf. (2.3)). Moreover, for $|u|<r_{1}$, we see that

$$
H(u)=\frac{1}{\tau} \int_{0}^{\epsilon|u|^{2}} \tau_{G}\left(\sqrt{\frac{c}{\epsilon}}, 0\right) d c=\frac{1}{\tau} \int_{0}^{\epsilon|u|^{2}} \frac{\pi}{\epsilon} d c=\frac{\pi}{\tau}|u|^{2},
$$

so that $H(u)$ fulfills (2.4) with $r=r_{1}$, as well. Accordingly, we can apply Proposition 2.2 to produce the symplectic diffeomorphism $\Lambda_{H}$ of the plane onto itself.

For further convenience, observe that $H(u)=F(u)$ for $|u|>r_{2}$, so that there exists $r^{*}>0$ such that $\Lambda_{H}^{-1}(v)=\Lambda_{F}^{-1}(v)$ for $|v| \geq r^{*}$. Indeed, it suffices to take $\varphi^{*}(t)$ solving $J u^{\prime}=\nabla H(u)$, with $\left|\varphi^{*}(t)\right| \geq r_{2}$ for every $t \in[0, \tau]$ (thus solving also $J u^{\prime}=\nabla F(u)$ ) and set $r^{*}=\sqrt{\frac{\pi}{\tau} H\left(\varphi^{*}(t)\right)}$. This fact has two important consequences:

- it holds

$$
\Lambda_{H}^{-1}(\lambda v)=\lambda \Lambda_{H}^{-1}(v), \quad \text { for every }|v| \geq r^{*}, \lambda>1 ;
$$

- we have

$$
\left\|\left(\Lambda_{H}^{-1}\right)^{\prime}(v)\right\| \leq L, \quad \text { for every } v \in \mathbb{R}^{2},
$$

for a suitable constant $L>0$.

Both claims follow from the fact that $\Lambda_{F}(u)$ is positively 1-homogeneous, since $\theta(u)$ is 0-homogeneous (cf. Remark 2.3).

For $u \in \mathbb{R}^{2}$, we set $S(u)=F(u)-H(u)$; moreover, we define, for $v \in \mathbb{R}^{2}$ and $t \in[0, T]$,

$$
\widetilde{H}(v)=H\left(\Lambda_{H}^{-1}(v)\right), \quad \widetilde{S}(v)=S\left(\Lambda_{H}^{-1}(v)\right), \quad \widetilde{R}(t, v)=R\left(t, \Lambda_{H}^{-1}(v)\right) .
$$

With these positions, system (1.6) is changed, via $\Lambda_{H}$, into

$$
J v^{\prime}=\nabla \widetilde{H}(v)+\nabla \widetilde{S}(v)+\nabla \widetilde{R}(t, v),
$$

that is, using (2.5) and (1.9),

$$
J v^{\prime}=\frac{2 N \pi}{T} v+\nabla \widetilde{S}(v)+\nabla \widetilde{R}(t, v) .
$$

We now claim that we are in the setting of Theorem 2.5; precisely,

- there exists $\widetilde{M}>0$ such that, for every $t \in[0, T]$ and every $v \in \mathbb{R}^{2}$,

$$
|\nabla \widetilde{R}(t, v)+\nabla \widetilde{S}(v)| \leq \widetilde{M}\left(1+|v|^{\alpha}\right)
$$


- $\quad$ denoting by $\mathfrak{S}$ the set of the $T$-periodic solutions to $J v^{\prime}=\frac{2 N \pi}{T} v$, it holds that

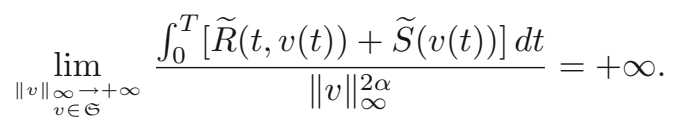

For the first claim, we observe preliminarily that, applying the Mean Value Theorem for $C^{1}$-maps on convex subsets of $\mathbb{R}^{2}$ to $\Lambda_{H}^{-1},(3.3)$ and $\Lambda_{H}^{-1}(0)=0$ we have

$$
\left|\Lambda_{H}^{-1}(v)\right| \leq L|v|, \quad \text { for every } v \in \mathbb{R}^{2} .
$$

From (1.10), (3.3) and (3.5), we obtain

$$
\begin{aligned}
|\nabla \widetilde{R}(t, v)| & =\left|\left[\left(\Lambda_{H}^{-1}\right)^{\prime}(v)\right]^{t} \nabla R\left(t, \Lambda_{H}^{-1}(v)\right)\right| \leq L\left|\nabla R\left(t, \Lambda_{H}^{-1}(v)\right)\right| \\
& \leq L M\left(1+\left|\Lambda_{H}^{-1}(v)\right|^{\alpha}\right) \leq L M\left(1+L^{\alpha}|v|^{\alpha}\right),
\end{aligned}
$$

proving the claim since $\nabla \widetilde{S}(v)$ is bounded (indeed, $S(u)=0$ for $|u|>r_{2}$ ).

We now prove the second claim. Again in view of the boundedness of $\widetilde{S}(v)$, it is equivalent to show that

$$
\lim _{\|v\|_{\substack{\infty \\ v \in \mathfrak{S}}}} \frac{\int_{0}^{T} \widetilde{R}(t, v(t)) d t}{\|v\|_{\infty}^{2 \alpha}}=+\infty
$$

To this aim, notice that

$$
v(t) \in \mathfrak{S} \Longleftrightarrow v(t)=\widetilde{\lambda} \psi(t+\widetilde{\theta}),
$$

for suitable positive constants $\tilde{\lambda}>0, \widetilde{\theta} \in[0, \tau[$, where

$$
\psi(t)=\left(\cos \left(\frac{2 N \pi}{T} t\right),-\sin \left(\frac{2 N \pi}{T} t\right)\right) .
$$

In particular, it turns out that $\|v\|_{\infty}=\widetilde{\lambda}$, so that (3.6) is equivalent to

$$
\lim _{\widetilde{\lambda} \rightarrow+\infty} \frac{1}{\widetilde{\lambda}^{2 \alpha}} \int_{0}^{T} \widetilde{R}(t, \widetilde{\lambda} \psi(t+\widetilde{\theta})) d t=+\infty, \quad \text { uniformly in } \widetilde{\theta} \in[0, \tau[.
$$

We now observe that, in view of (3.2) and the position of $r^{*}$, for $\tilde{\lambda} \geq r^{*}$ we have

$$
\begin{aligned}
\Lambda_{H}^{-1}(\widetilde{\lambda} \psi(t+\widetilde{\theta})) & =\Lambda_{H}^{-1}\left(\frac{\tilde{\lambda}}{r^{*}} r^{*} \psi(t+\widetilde{\theta})\right) \\
& =\frac{\widetilde{\lambda}}{r^{*}} \Lambda_{H}^{-1}\left(r^{*} \psi(t+\widetilde{\theta})\right)=\frac{\widetilde{\lambda}}{r^{*}} \varphi^{*}\left(t+\theta^{*}(\widetilde{\theta})\right) .
\end{aligned}
$$

Since, as remarked before, $\varphi^{*}(t)$ satisfies (1.8), there exist $\rho>0$ and $\theta \in[0, \tau[$ (depending on $\varphi^{*}(t)$ and $\theta^{*}(\widetilde{\theta})$ ) such that

$$
\varphi^{*}\left(t+\theta^{*}(\widetilde{\theta})\right)=\rho \varphi(t+\theta) .
$$


Summing up, we have

$$
\begin{aligned}
\frac{1}{\widetilde{\lambda}^{2 \alpha}} \int_{0}^{T} \widetilde{R}(t, \widetilde{\lambda} \psi(t+\widetilde{\theta})) d t & =\frac{1}{\widetilde{\lambda}^{2 \alpha}} \int_{0}^{T} R\left(t, \Lambda_{H}^{-1}(\widetilde{\lambda} \psi(t+\widetilde{\theta}))\right) d t \\
& =\frac{1}{\widetilde{\lambda}^{2 \alpha}} \int_{0}^{T} R\left(t, \widetilde{\lambda} \frac{\rho}{r^{*}} \varphi(t+\theta)\right) d t,
\end{aligned}
$$

so that we conclude in view of (1.11).

A couple of remarks about Theorem 1.1 are now in order.

Remark 3.1. When $\alpha=0$ in (1.10), i.e., when $\nabla R(t, u)$ is bounded, (1.11) reads as

$$
\lim _{\lambda \rightarrow+\infty} \int_{0}^{T} R(t, \lambda \varphi(t+\theta)) d t=+\infty, \quad \text { uniformly in } \theta \in\left[0, \frac{T}{N}[.\right.
$$

Bounded perturbations of resonant problems represent the setting where the Ahmad-Lazer-Paul condition was originally introduced $[1,23]$.

We also point out that, according to [15, Theorem 1.1], the conclusion of Theorem 1.1 still holds true if (1.11) is replaced by

$$
\lim _{\lambda \rightarrow+\infty} \frac{1}{\lambda^{2 \alpha}} \int_{0}^{T} R(t, \lambda \varphi(t+\theta)) d t=-\infty, \quad \text { uniformly in } \theta \in\left[0, \frac{T}{N}[.\right.
$$

Remark 3.2. Our choice to study perturbations of positively 2-homogeneous Hamiltonians in Theorem 1.1 is mainly motivated by the fact that, in this setting, (3.3) holds true. As a consequence, one easily gets relation (3.4), assuming the corresponding bound (1.10) for the growth of $\nabla R(t, u)$.

However, some generalizations to perturbations of other isochronous centers are possible. For instance, as in [18], one can consider the scalar $p$-Laplacian equation $(p>1)$

$$
\left(\left|x^{\prime}\right|^{p-2} x^{\prime}\right)^{\prime}+\mu|x|^{p-2} x^{+}-\nu|x|^{p-2} x^{-}+w(t, x)=0,
$$

whose associated Hamiltonian $F(x, y)=\frac{1}{p}\left(\mu\left(x^{+}\right)^{p}+\nu\left(x^{-}\right)^{p}\right)+\frac{1}{q}|y|^{q}$ (with $q$ given by $\frac{1}{p}+\frac{1}{q}=1$ ) is not positively homogeneous for $p \neq 2$, but gives birth to an isochronous center. In this case, even if (3.3) is not fulfilled, a suitable growth assumption on $w(t, x)$, depending on $p$, ensures the validity of (3.4), with $\alpha=0$.

We conclude the section focusing on some consequences of Theorem 1.1 for the scalar equation (1.4) and for related problems.

Proof of Corollary 1.2. Set $u=(x, y), F(u)=\frac{1}{2}\left(\mu\left(x^{+}\right)^{2}+\nu\left(x^{-}\right)^{2}+\right.$ $\left.y^{2}\right), R(t, u)=(W(t, x), 0)$, and $\varphi(t)=\sqrt{\mu}\left(\phi\left(t+\frac{\pi}{2 \sqrt{\mu}}\right), \phi^{\prime}\left(t+\frac{\pi}{2 \sqrt{\mu}}\right)\right)$. The thesis follows plainly from Theorem 1.1, observing that (1.5) implies (1.9), (1.12) implies (1.10) and (1.13) implies (1.11). tor"

This situation can be extended to the perturbed "bi-asymmetric oscilla-

$$
\left\{\begin{array}{l}
x^{\prime}=\mu_{1} y^{+}-\nu_{1} y^{-}+w_{1}(t, y) \\
y^{\prime}=-\mu_{2} x^{+}+\nu_{2} x^{-}-w_{2}(t, x)
\end{array}\right.
$$

being 
- $\mu_{1}, \mu_{2}, \nu_{1}, \nu_{2}>0$ such that, for a positive integer $N$,

$$
\frac{\pi}{2}\left(\frac{1}{\sqrt{\mu_{1} \mu_{2}}}+\frac{1}{\sqrt{\mu_{1} \nu_{2}}}+\frac{1}{\sqrt{\nu_{1} \nu_{2}}}+\frac{1}{\sqrt{\mu_{2} \nu_{1}}}\right)=\frac{T}{N} ;
$$

- $\quad w_{1}, w_{2}:[0, T] \times \mathbb{R} \rightarrow \mathbb{R}$ continuous functions satisfying, for suitable constants $M>0, \alpha \in[0,1[$,

$$
\left|w_{i}(t, z)\right| \leq M\left(1+|z|^{\alpha}\right), \quad \text { for every } t \in[0, T], z \in \mathbb{R}, i=1,2 .
$$

System (3.8) was already considered, for instance, in [10,28], including the asymmetric equation (1.4) (for $\mu_{1}=\nu_{1}=1, w_{1}(t, y) \equiv 0$ ). When (3.9) is fulfilled, every nontrivial solution to the autonomous system

$$
\left\{\begin{array}{l}
x^{\prime}=\mu_{1} y^{+}-\nu_{1} y^{-} \\
y^{\prime}=-\mu_{2} x^{+}+\nu_{2} x^{-},
\end{array}\right.
$$

is $T$-periodic; fixed the solution $\varphi(t)=\left(\varphi_{1}(t), \varphi_{2}(t)\right)$ such that $\varphi(0)=(1,0)$, every other (nontrivial) one has the form $\lambda \varphi(t+\theta)$ for suitable constants $\lambda>0, \theta \in\left[0, \frac{T}{N}[\right.$. According to Theorem 1.1, the planar system (3.8) has a $T$-periodic solution if the following condition is fulfilled:

uniformly in $\theta \in\left[0, \frac{T}{N}[\right.$, it holds

$$
\lim _{\lambda \rightarrow+\infty} \frac{1}{\lambda^{2 \alpha}} \int_{0}^{T}\left[W_{1}\left(t, \lambda \varphi_{1}(t+\theta)\right)+W_{2}\left(t, \lambda \varphi_{2}(t+\theta)\right)\right] d t=+\infty,
$$

being $W_{i}(t, z)=\int_{0}^{z} w_{i}(t, \xi) d \xi, i=1,2$.

\section{Comparing Landesman-Lazer and Ahmad-Lazer-Paul conditions}

In this section, we compare the Ahmad-Lazer-Paul condition (1.11) with the planar version of the Landesman-Lazer one, given in [10].

For simplicity, we will limit ourselves to the equation

$$
J u^{\prime}=\nabla F(u)+\nabla R(t, u),
$$

with $\nabla R(t, u)$ a bounded function. Accordingly, we are interested in condition (3.7), written in the equivalent way

$$
\lim _{\lambda \rightarrow+\infty} \int_{0}^{T} R(t, \lambda \varphi(t+\theta)) d t=+\infty, \quad \text { uniformly in } \theta \in[0, T],
$$

being $\varphi(t)$ fixed as in Sect. 3. We recall that, in this setting, the planar Landesman-Lazer condition [10] reads as follows:

for every $\theta \in[0, T]$, it holds

$$
\int_{0}^{T} \liminf _{\substack{\lambda \rightarrow+\infty \\ \omega \rightarrow \theta}}\langle\nabla R(t, \lambda \varphi(t+\omega)) \mid \varphi(t+\omega)\rangle d t>0 .
$$

Our result is the following, in connection with [11, Theorem 2.1] (dealing with second order equations and some abstract variants).

Theorem 4.1. Condition (4.2) implies (4.1).

To prove the theorem, we state a preliminary lemma. 
Lemma 4.2. Assume (4.2). Then, there exist $\lambda_{0}>0, \theta_{1}, \ldots, \theta_{j} \in[0, T], \delta_{1}, \ldots$, $\delta_{j}>0$ and $h_{1}, \ldots, h_{j} \in L^{1}(0, T)$, with $\int_{0}^{T} h_{i}(t) d t>0$ for every $i=1, \ldots, j(j$ being a suitable integer), such that

$$
\bigcup_{i=1}^{j}\left[\theta_{i}-\delta_{i}, \theta_{i}+\delta_{i}\right] \supset[0, T]
$$

and, for every $i=1, \ldots, j$ and every $t \in[0, T]$,

$$
\langle\nabla R(t, \lambda \varphi(t+\omega)) \mid \varphi(t+\omega)\rangle \geq h_{i}(t), \quad \text { if }\left|\omega-\theta_{i}\right| \leq \delta_{i}, \lambda \geq \lambda_{0} .
$$

Proof. For simplicity, we set $l_{\kappa}(t, \omega)=\langle\nabla R(t, \kappa \varphi(t+\omega)) \mid \varphi(t+\omega)\rangle$.

Fix $\hat{\theta} \in[0, T]$. In view of the definition of inferior limit and Fatou's lemma, we have

$$
\liminf _{\substack{\lambda \rightarrow+\infty \\ \delta \rightarrow 0}} \int_{0}^{T} \inf _{\substack{\kappa \geq \lambda,|\omega-\hat{\theta}| \leq \delta \\ \kappa(n,}} l_{\kappa}(t, \omega) d t>0 .
$$

Therefore, there exist $\lambda_{0}=\lambda_{0}(\hat{\theta}) \geq 1, \delta_{0}=\delta_{0}(\hat{\theta})>0$ such that

$$
\int_{0}^{T} \inf _{\kappa \geq \lambda_{0},|\omega-\hat{\theta}| \leq \delta_{0}} l_{\kappa}(t, \omega) d t>0 .
$$

We set

$$
h(t, \hat{\theta})=\inf _{\kappa \geq \lambda_{0},|\omega-\hat{\theta}| \leq \delta_{0}} l_{\kappa}(t, \omega)
$$

of course, $\int_{0}^{T} h(t, \hat{\theta}) d t>0$ and, by the definition, $l_{\kappa}(t, \omega) \geq h(t, \hat{\theta})$ for every $t \in[0, T], \kappa \geq \lambda_{0}(\hat{\theta})$ and $|\omega-\hat{\theta}| \leq \delta_{0}(\hat{\theta})$. Repeating the argument for every $\hat{\theta} \in[0, T]$ and using the compactness of $[0, T]$, there exist $\theta_{1}, \ldots, \theta_{j} \in[0, T], \delta_{1}\left(\theta_{1}\right), \ldots, \delta_{j}\left(\theta_{j}\right)>0$ such that (4.3) holds true. Setting

$$
\lambda_{0}=\max _{i=1, \ldots, j}\left\{\lambda_{0}\left(\theta_{i}\right)\right\}, \quad h_{i}(t)=h\left(t, \theta_{i}\right), \quad i=1, \ldots, j,
$$

we finally get (4.4).

Since the converse statement is easily seen to hold true, Lemma 4.2 can be viewed as a characterization of condition (4.2) (cf., as a comparison term, [11, Proposition 3.1]).

Proof of Theorem 4.1. Without loss of generality, we can assume $R(t, 0) \equiv 0$; moreover, as in the proof of Lemma 4.2, we set

$$
l_{\kappa}(t, \theta)=\langle\nabla R(t, \kappa \varphi(t+\theta)) \mid \varphi(t+\theta)\rangle .
$$


For every $t \in[0, T], \lambda \geq \lambda_{0}$ and $\theta \in[0, T]$, we have

$$
\begin{aligned}
R(t, \lambda \varphi(t+\theta)) & =\int_{0}^{1} \frac{d}{d \kappa} R(t, \kappa \lambda \varphi(t+\theta)) d \kappa \\
& =\lambda \int_{0}^{1}\langle\nabla R(t, \kappa \lambda \varphi(t+\theta)) \mid \varphi(t+\theta)\rangle d \kappa=\int_{0}^{\lambda} l_{\kappa}(t, \theta) d \kappa \\
& =\int_{0}^{\lambda_{0}} l_{\kappa}(t, \theta) d \kappa+\int_{\lambda_{0}}^{\lambda} l_{\kappa}(t, \theta) d \kappa \\
& \geq-\lambda_{0} \max _{t, \theta \in[0, T], \kappa \in\left[0, \lambda_{0}\right]}\left|l_{\kappa}(t, \theta)\right|+\int_{\lambda_{0}}^{\lambda} h_{i}(t) d \kappa,
\end{aligned}
$$

being the index $i$ such that $\theta \in\left[\theta_{i}-\delta_{i}, \theta_{i}+\delta_{i}\right]$ (keeping the notation of Lemma $4.2)$. Integrating on $[0, T]$ we obtain

$$
\begin{aligned}
\int_{0}^{T} R(t, \lambda \varphi(t+\theta)) d t & \geq-C_{1}+\left(\lambda-\lambda_{0}\right) \int_{0}^{T} h_{i}(t) d t \\
& \geq-C_{1}+\left(\lambda-\lambda_{0}\right) \min _{i=1, \ldots, j} \int_{0}^{T} h_{i}(t) d t
\end{aligned}
$$

being $C_{1}=T \lambda_{0} \max _{t, \theta \in[0, T], \kappa \in\left[0, \lambda_{0}\right]}\left|l_{\kappa}(t, \theta)\right|$. The conclusion follows, since, for $i=1, \ldots, j, \int_{0}^{T} h_{i}(t) d t>0$ in view of Lemma 4.2 .

Remark 4.3. In view of (4.5), the same arguments show that, when (1.10) is satisfied for $\alpha \in[0,1 / 2[$, condition (4.2) still implies (1.11). In such a case, however, in order for the integral in (4.2) to make sense, one has to assume that, for a suitable $\eta \in L^{1}(0, T)$, it holds

$$
\langle\nabla R(t, \lambda u) \mid u\rangle \geq \eta(t), \quad \text { for every } t \in[0, T],|u| \leq 1, \lambda \geq 1 .
$$

\section{Acknowledgments}

We are grateful to Alessandro Fonda for having suggested this research, as well as for several useful discussions. We also thank the referee for some suggestions which helped us to make the paper more readable.

\section{Appendix}

We now discuss some regularity issues about the functions used in Sect. 2, keeping the notation used therein. For functions depending on $u \in \mathbb{R}^{2}$, we will write $\partial_{i}, i=1,2$, to denote the partial derivative with respect to the $i$-th component of $u$. Preliminarily, we observe that the map

$$
\mathbb{R} \times \mathbb{R}_{*}^{2} \ni(t, u) \mapsto \zeta(t, u)=\left(\zeta_{1}(t, u), \zeta_{2}(t, u)\right) \in \mathbb{R}_{*}^{2}
$$

is of class $C^{1}$. 
Regularity of $u \mapsto \tau(u)$.

As in Sect. 3, for $c \in \mathbb{R}_{*}^{+}$we define $\xi(c)$ as the unique positive number such that $H(\xi(c), 0)=c$. The map $c \mapsto \xi(c)$ is continuous and the Implicit Function Theorem ensures that it is of class $C^{1}$. Indeed,

$$
\left.\frac{\partial}{\partial d}(H(d, 0)-c)\right|_{d=\xi(c)}=\partial_{1} H(\xi(c), 0)=\frac{1}{\xi(c)}\langle\nabla H(\xi(c), 0) \mid(\xi(c), 0)\rangle \neq 0 .
$$

Next, for $x \in \mathbb{R}_{*}^{+}$, we define $\Pi(x)$ as the second strictly positive real number such that $\zeta_{2}(\Pi(x),(x, 0))=0$. Since all the nontrivial solutions to $J u^{\prime}=$ $\nabla H(u)$ are periodic and describe, in the clockwise sense, a strictly star-shaped Jordan curve, $\Pi(x)$ is the period of the orbit passing through $(x, 0)$. Since $\nabla H(u) \neq 0$ for every $u \neq 0,[17,(\mathrm{v})$, p. 83$]$ ensures that $\Pi(x)$ is continuous. We claim that it is actually of class $C^{1}$. Indeed, using again the Implicit Function Theorem,

$$
\begin{aligned}
\left.\frac{\partial}{\partial t} \zeta_{2}(t,(x, 0))\right|_{t=\Pi(x)} & =-\partial_{1} H(\zeta(\Pi(x),(x, 0))) \\
& =-\frac{\langle\nabla H(\zeta(\Pi(x),(x, 0))) \mid \zeta(\Pi(x),(x, 0))\rangle}{\zeta_{1}(\Pi(x),(x, 0))} \neq 0 .
\end{aligned}
$$

Since $\tau(u)=\Pi(\xi(H(u)))$, for $u \in \mathbb{R}_{*}^{2}$, we conclude.

Regularity of $u \mapsto \theta(u)$.

$\left.\overline{\text { Let us fix } u^{*} \in \mathbb{R}_{*}^{2} \backslash\left(\mathbb{R}_{*}^{+}\right.} \times\{0\}\right)$. The continuity of $\theta(u)$ in $u^{*}$ follows from the continuity of $\zeta(t, u)$.

We now prove that $\theta(u)$ is of class $C^{1}$ in a neighborhood of $u^{*}$. By the Implicit Function Theorem, repeating a similar argument as before, we deduce that there exist:

- a neighborhood $\mathcal{U}$ of $u^{*}$ and a neighborhood $\mathcal{T}$ of $\frac{\tau}{2 \pi} \theta\left(u^{*}\right) ;$

- a $C^{1}$-map $t: \mathcal{U} \rightarrow \mathcal{T}$,

such that

$$
\zeta_{2}(-t, u)=0, \quad(t, u) \in \mathcal{T} \times \mathcal{U} \quad \Longleftrightarrow \quad t=t(u) .
$$

Since $\theta(u)$ is continuous at $u^{*}$, we get $t(u)=\frac{\tau}{2 \pi} \theta(u)$ for $u$ in a neighborhood of $u^{*}$, getting the desired conclusion.

We now examine the regularity in a point $u_{0} \in \mathbb{R}_{*}^{+} \times\{0\}$. As before, one can construct locally the function $t(u)$ satisfying (A.1). By the definition of $\theta(u) \in\left[0,2 \pi\left[\right.\right.$, we can infer that, for $u=\left(u_{1}, u_{2}\right)$ in a neighborhood of $u_{0}$,

$$
\theta(u)= \begin{cases}\frac{2 \pi}{\tau} t(u) & \text { for } u_{2}<0 \\ \frac{2 \pi}{\tau}(t(u)+\tau) & \text { for } u_{2} \geq 0 .\end{cases}
$$

From (A.2), we deduce both the fact that $\nabla \theta(u)$ extends to a continuous function on the whole $\mathbb{R}_{*}^{2}$ and the fact that the map $\mathbb{R}_{*}^{2} \ni u \mapsto(\cos \theta(u),-\sin \theta(u))$ is continuous.

We lastly discuss the differentiability of $u \mapsto(\cos \theta(u),-\sin \theta(u))$. Of course, we only need to focus on $u_{0} \in \mathbb{R}_{*}^{+} \times\{0\}$; for simplicity, moreover, 
we just consider the map $u \mapsto \cos \theta(u)$. The existence in $u_{0}$ and the continuity in a neighborhood of $u_{0}$ are ensured for $\partial_{2} \cos (\theta(u))$, since $\nabla \theta(u)$ exists out of $\mathbb{R}_{*}^{+} \times\{0\}$ and can be extended to the whole $\mathbb{R}_{*}^{2}$. For what concerns $\partial_{1} \cos \theta(u)$, we have, since $\theta(u) \equiv 0$ on $\mathbb{R}_{*}^{+} \times\{0\}$,

$$
\left.\partial_{1} \cos \theta(u)\right|_{u=u_{0}}=\lim _{\delta \rightarrow 0} \frac{\cos \theta\left(u_{0}+(\delta, 0)\right)-\cos \theta\left(u_{0}\right)}{\delta}=0,
$$

so that the existence in $u_{0}$ is guaranteed. As for the continuity in a neighborhood of $u_{0}$, observe that, for $u \notin \mathbb{R}_{*}^{+} \times\{0\}$, one has

$$
\begin{aligned}
\lim _{u \rightarrow u_{0}} \partial_{1} \cos \theta(u) & =-\lim _{u \rightarrow u_{0}} \sin \theta(u) \partial_{1} \theta(u) \\
& =-\frac{2 \pi}{\tau} \lim _{u \rightarrow u_{0}} \sin \theta(u) \partial_{1} t(u)=-\frac{2 \pi}{\tau} \sin \theta\left(u_{0}\right) \partial_{1} t\left(u_{0}\right)
\end{aligned}
$$

The conclusion follows from the fact that, since $t(u) \equiv 0$ on $\mathbb{R}_{*}^{+} \times\{0\}$, it holds $\left\langle\nabla t\left(u_{0}\right) \mid u_{0}\right\rangle=0$.

\section{References}

[1] Ahmad, S., Lazer, A.C., Paul, J.L.: Elementary critical point theory and perturbations of elliptic boundary value problems at resonance, Indiana Univ. Math. J. 25, 933-944 (1976)

[2] Bartsch, T., Szulkin, A.: Hamiltonian systems: periodic and homoclinic solutions by variational methods. In: Handbook of Differential Equations: Ordinary Differential Equations, vol. II. pp. 77-146. Elsevier, Amsterdam (2005)

[3] Bereanu, C., Jebelean, P., Mawhin, J.: Variational methods for nonlinear perturbations of singular $\phi$-Laplacians. Atti Accad. Naz. Lincei Cl. Sci. Fis. Mat. Natur. Rend. Lincei (9) Mat. Appl. 22, 89-111 (2011)

[4] Bonheure, D., Fabry, C.: A variational approach to resonance for asymmetric oscillators. Commun. Pure Appl. Anal. 6, 163-181 (2007)

[5] Dancer, E.N.: Boundary-value problems for weakly nonlinear ordinary differential equations. Bull. Aust. Math. Soc. 15, 321-328 (1976)

[6] Daouas, A., Timoumi, M.: Subharmonics for not uniformly coercive Hamiltonian systems. Nonlinear Anal. 66, 571-581 (2007)

[7] Fabry, C.: Landesman-Lazer conditions for periodic boundary value problems with asymmetric nonlinearities. J. Differ. Equ. 116, 405-418 (1995)

[8] Fabry, C., Fonda, A.: Periodic solutions of perturbed isochronous Hamiltonian systems at resonance. J. Differ. Equ. 214, 299-325 (2005)

[9] Fonda, A.: Positively homogeneous Hamiltonian systems in the plane. J. Differ. Equ. 200, 162-184 (2004) 
[10] Fonda, A., Garrione, M.: Double resonance with Landesman-Lazer conditions for planar systems of ordinary differential equations. J. Differ. Equ. 250, 10521082 (2011)

[11] Fonda, A., Garrione, M.: Nonlinear resonance: a comparison between Landesman-Lazer and Ahmad-Lazer-Paul conditions. Adv. Nonlinear Stud. 11, 391$404(2011)$

[12] Fonda, A., Mawhin, J.: Planar differential systems at resonance. Adv. Differ. Equ. 11, 1111-1133 (2006)

[13] Fonda, A., Sabatini, M., Zanolin, F.: Periodic solutions of perturbed Hamiltonian systems in the plane by the use of the Poincaré-Birkhoff theorem. Topol. Methods Nonlinear Anal. (2012, to appear)

[14] Fučik, S.: Boundary value problems with jumping nonlinearities. Časopis Pěst. Mat. 101, 69-87 (1976)

[15] Han, Z.-Q.: Existence of periodic solutions of linear Hamiltonian systems with sublinear perturbation. Bound. Value Probl. 2010, 1-12 (2010)

[16] Han, Z.-Q., Li, Q.: Existence of critical point for abstract resonant problems with unbounded nonlinearities and applications to differential equations. Nonlinear Anal. 65, 1654-1668 (2006)

[17] Henrard, M., Zanolin, F.: Bifurcation from a periodic orbit in perturbed planar Hamiltonian systems. J. Math. Anal. Appl. 277, 79-103 (2003)

[18] Jiang, M.-Y.: A Landesman-Lazer type theorem for periodic solutions of the resonant asymmetric p-Laplacian equation. Acta Math. Sinica 21, 1219-1228 (2005)

[19] Jiang, M.-Y.: Periodic solutions of some resonant Hamiltonian systems. J. Differ. Equ. 244, 2156-2184 (2008)

[20] Landesman, E., Lazer, A.C.: Nonlinear perturbations of linear elliptic boundary value problems at resonance. J. Math. Mech. 19, 609-623 (1970)

[21] Lazer, A.C., Leach, D.E.: Bounded perturbations of forced harmonic oscillators at resonance. Ann. Mat. Pura Appl. 82, 49-68 (1969)

[22] Liu, J.Q.: A generalized saddle point theorem. J. Differ. Equ. 82, 372-385 (1989)

[23] Mawhin, J., Willem, M.: Critical Point Theory and Hamiltonian Systems. Applied Mathematical Sciences, vol. 74. Springer, New York (1989)

[24] Rabinowitz, P.: Minimax Methods in Critical Point Theory with Applications to Differential Equations. CBMS Regional Conference Series in Mathematics, vol. 65. AMS, Providence (1986)

[25] Schechter, M.: Resonance problems with respect to Fučik spectrum. Trans. Am. Math. Soc. 352, 4195-4205 (2000)

[26] Szulkin, A.: A relative category and applications to critical point theory for strongly indefinite functionals. Nonlinear Anal. 15, 725-739 (1990) 
[27] Tang, C.L.: Periodic solutions for nonautonomous second order systems with sublinear nonlinearity. Proc. Am. Math. Soc. 126, 3263-3270 (1998)

[28] Yang, X.: Unbounded solutions of a class of planar systems. J. Math. Anal. Appl. 296, 708-718 (2004)

Alberto Boscaggin and Maurizio Garrione SISSA, International School for Advanced Studies Via Bonomea, 265

34136 Trieste

Italy

e-mail: boscaggi@sissa.it

Maurizio Garrione

e-mail: garrione@sissa.it

Received: 21 February 2012.

Accepted: 19 June 2012. 